\title{
Compositional Mapping by Z-Contrast Imaging
}

\author{
M.A. Roldam ${ }^{1,2}$, D. Hernandez-Maldonado ${ }^{1}$, J. Hernandez-Saz ${ }^{1}$, M. Herrera ${ }^{1}$, M.P. Guerrero ${ }^{3}$, \\ P.L. Galindo ${ }^{3}$, D. Alonso-Alvarez ${ }^{4}$, J.M. Ripalda ${ }^{4}$, B. Alen ${ }^{4}$, Y. Gonzalez ${ }^{4}$, M. Varela ${ }^{2}$, \\ S.J. Pennycook ${ }^{2}$, S. I. Molina ${ }^{1}$ \\ ${ }^{1}$ Departamento de Ciencia de los Materiales e I.M. y Q.I., Facultad de Ciencias, Universidad de \\ Cádiz, Campus Río San Pedro, s/n, 11510 Puerto Real, Cádiz, Spain \\ ${ }^{2}$ Materials Science and Technology Division, Oak Ridge National Laboratory, Oak Ridge, TN 37831 \\ ${ }^{3}$ Departamento de Lenguajes y Sistemas Informáticos, Universidad de Cádiz, Campus Río San Pedro, \\ 11510 Puerto Real, Cádiz, Spain \\ ${ }^{4}$ Instituto de Microelectronica de Madrid (CNM, CSIC), Isaac Newton 8, 28760 Tres Cantos, Madrid, \\ Spain
}

Quantitative compositional information can be extracted from high-resolution Z-contrast images by comparison of simulated and experimental images. We developed a phenomenological method to determine quantitatively the composition of a material with atomic column spatial resolution directly from the analysis of local integrated intensities of aberration-corrected Z-contrast experimental images [1]. In this work we apply this method to high-resolution aberration-corrected Z-contrast images acquired at $100 \mathrm{kV}$ with a dedicated Nion UltraSTEM scanning transmission electron microscope, which is equipped with a Nion aberration-corrector and a Gatan Enfina EELS detector. Specimens for Z-contrast imaging were prepared by mechanical thinning and $\mathrm{Ar}^{+}$ion milling using a Precision Ion Polishing System (PIPS). A beam energy less than $3.5 \mathrm{kV}$ has been selected to reduce amorphisation of the sample. As a final step, the sample was introduced in a Fischione ion mill at $12^{\circ}$ and $0.5 \mathrm{kV}$ to reduce surface damage. The thickness of the analyzed region was determined from the analysis of the corresponding spatially resolved low-loss EELS signal [2].

In order to estimate the composition in each column, we follow a procedure previously published [1] in which the first step is to detect pixels with the local intensity maxima associated; for that purpose we apply the Peak Pairs software [3]. Once these maxima intensity pixels are located, it is straightforward, with the help of the image processing software, to measure the intensities integrated within a selected area of the projected unit cell. In order to decide the best integration area, several measurements have been taken using different integration areas. A certain area of integration was finally selected that gives normalized integrated intensity ratios $R$ as shown in equation (1). $R$ values were determined following the procedure described in ref. [1], but taking whole unit cells in the present study. $R$ values show some advantages when they are defined in this way: (a) $R$ depends almost linearly on the composition, (b) $R$ has a very low dependence on specimen thickness over a convenient thickness range, (c) $\mathrm{R}$ values are almost unaffected by surrounding dumbbells so that the signal is essentially due to just the atoms contained within the selected atomic column.

$$
\mathrm{R}_{\mathrm{i}}=\mathrm{I}_{\text {column }} / \mathrm{I}_{\text {substrate }}
$$


We have verified that R-values can be considered almost independent of the specimen thickness over a convenient range. We have demonstrated that there is a linear regression equation to relate $\mathrm{R}$ with the composition $\mathrm{x}$ in each atomic column [1].

Applying this method to a real image, it is possible to make an elemental map from a selected area of a HAADF image. In this work, we study a layer of $1.5 \mathrm{~nm}$ of $\mathrm{GaAs}_{0.62} \mathrm{P}_{0.38}$ (nominal composition), that is working like a strain compensation layer (SCL) in a sample containing quantum dots of InAs on a GaAs substrate. The studied region is $60 \pm 5 \mathrm{~nm}$ thick.

We analyze the homogeneity of P distribution along the entire SCL. In order to verify this, the region marked with white lines in Fig. 1a was studied following the procedure described above. The experimental fitting equation used in this study was:

$$
\mathrm{R}_{\mathrm{i}}=1+0.01475 x_{i}
$$

Fig. 1b shows with colored dots the composition values obtained from equation (2), assuming that the region outside the SCL that shows the maximum $\mathrm{R}_{\mathrm{i}}$ values corresponds to a $0 \%$ content of $\mathrm{P}$ atoms, therefore to As columns in pure GaAs. Looking at Fig. 1b, we can see in the colored chemical map that there is no homogeneity in the SCL; therefore there is variation in the composition of $\mathrm{P}$ along the entire SCL.

\section{References}

[1] S. I. Molina et al., Ultramicroscopy 109, 172 (2009); erratum: 109 (2009) 1315.

[2] R. F. Egerton, Electron Energy-Loss Spectroscopy in the Electron Microscope; Plenum: New York, 1986.

[3] P. L. Galindo et al., Ultramicroscopy 107, 1186 (2007).

[4] This research was sponsored by the Office of Basic Energy Sciences, Materials Sciences and Engineering Division, U.S. Department of Energy (SJP, MV), by the Spanish MCI (projects CONSOLIDER INGENIO 2010 CSD2009-00013 andTEC2008-06756-C03-02/TEC,) and the Junta de Andalucía (PAI research's groups TEP-120 and TIC-145; project P08-TEP-03516).
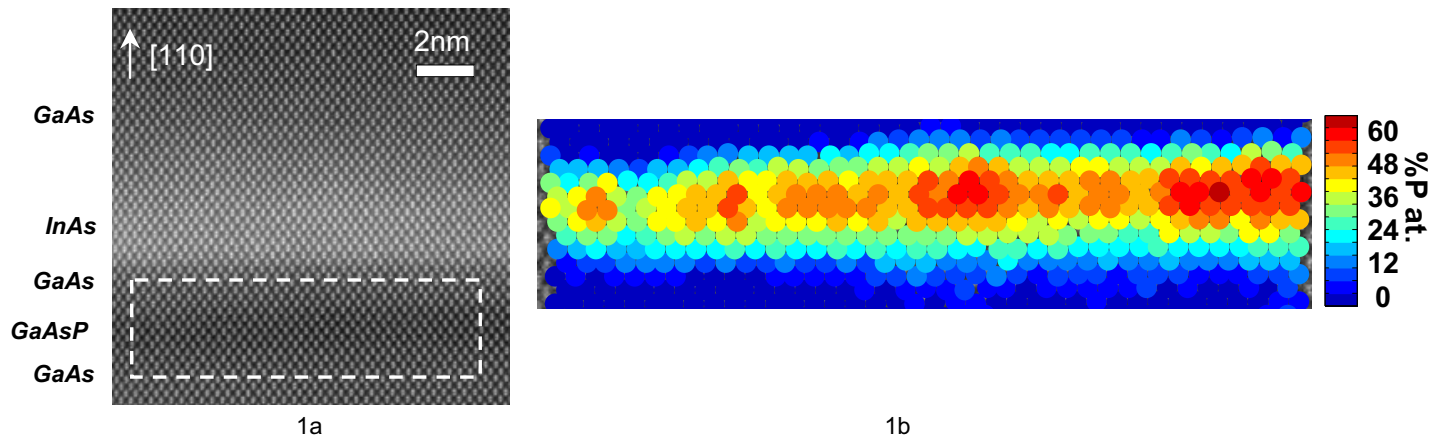

Fig. 1 (a) HAADF image of an InAs Quantum Dot grown on a GaAs substrate, with a SCL of $\mathrm{GaAs}_{1-\mathrm{x}} \mathrm{P}_{\mathbf{x}}$; (b) P elemental mapping in the SCL. 